\title{
Les Bols élaborés au tour d'Abu Hamid : rupture technique au 4 e millénaire avant J.-C. dans le Levant-Sud.
}

In: Paléorient. 1997, Vol. 23 №1. pp. 25-43.

\begin{abstract}
In the Southern Levant, during the 4th millennium BC, a new morpho-technical ceramic type appears : the bowls with a « V » profile. These bowls are present in the upper levels of the site of Abu Hamid. A technological analysis, both of surface features and microfabrics, has enabled us to reconstruct the manufacturing process, to identify centres of production and to propose hypotheses about their function. The bowls with a «V» profile were shaped on the wheel. The ones from Abu Hamid came from remote production centres, some in the Negev. Their function seems to have been cultual. Their distribution could reflect the emergence of a vast politico- cultic community in the Southern Levant at the beginning of the 4th millennium BC.
\end{abstract}

\section{Résumé}

Au 4e millénaire avant J.-C, dans le Levant-Sud, apparaît une nouvelle catégorie morpho-technique de récipients céramiques : les bols au profil en « V». Ces bols sont présents dans les niveaux supérieurs du site d'Abu Hamid. Une analyse technologique, portant simultanément sur les macro-traces et les micro -fabriques, permet de reconstituer la chaîne opératoire de fabrication, d'identifier les centres de production, et de proposer des hypothèses sur leur fonction et les facteurs à l'origine de leur fabrication. Les bols au profil en « V» étaient élaborés au tour. Les bols d'Abu Hamid provenaient de centres de production éloignés, dont le Negev. Leur fonction paraît avoir été d'ordre cultuel. Leur distribution marquerait la mise en place d'une vaste communauté politico-cultuelle dans le Levant-Sud au début du 4e millénaire avant J.-C.

Citer ce document / Cite this document :

Courty Marie-A., Roux Valentine. Les Bols élaborés au tour d'Abu Hamid : rupture technique au 4e millénaire avant J.-C. dans le Levant-Sud. In: Paléorient. 1997, Vol. 23 N¹. pp. 25-43.

doi : $10.3406 /$ paleo.1997.4643

http://www.persee.fr/web/revues/home/prescript/article/paleo_0153-9345_1997_num_23_1_4643 


\title{
LES BOLS ÉLABORÉS AU TOUR D'ABU HAMID : RUPTURE TECHNIQUE AU $4^{\mathrm{e}}$ MILLÉNAIRE AVANT J.-C. DANS LE LEVANT-SUD
}

\author{
V. ROUX et M.-A. COURTY
}

Résumé : Au $4^{e}$ millénaire avant J.-C., dans le Levant-Sud, apparaît une nouvelle catégorie morpho-lechnique de récipients céramiques: les bols au profil en " $V$ ". Ces bols sont présents dans les niveaux supérieurs du site d'Abu Hamid. Une analyse technologique, portant simultanément sur les macro-traces et les micro-fabriques, permet de reconstituer la chaîne opératoire de fabrication, d'identifier les centres de production, et de proposer des hypothèses sur leur fonction et les facteurs à l'origine de leur fabrication. Les bols au profil en " $V$ » étaient élaborés au tour. Les bols d'Abu Hamid provenaient de centres de production éloignés, dont le Negev. Leur fonction parait avoir été d'ordre cultuel. Leur distribution marquerait la mise en place d'une vaste communauté politico-cultuelle dans le Levant-Sud au début du $4^{e}$ millénaire avant J.-C.

Abstract: In the Southern Levant, during the 4th millennium BC, a new morpho-technical ceramic type appears: the bowls with $a$ "V" profile. These bowls are present in the upper levels of the site of Abu Hamid. A technological analvsis, both of surface features and microfabrics, has enabled us to reconstruct the manufacturing process, to identify centres of production and to propose hypotheses about their function. The bowls with $a$ "V" profile were shaped on the wheel. The ones from Abu Hamid came from remote production centres, some in the Negev. Their function seems to have been cultual. Their distribution could reflect the emergence of a vast politico-cultic community in the Southern Levant at the beginning of the 4th millennium BC.

Mots clefs : Céramique, Macro-traces, Micro-fabriques, Tour, Chalcolithique, Levant-Sud.

Key-words : Ceramics, Macro-traces, Micro-fabrics, Wheel, Chalcolithic, South-Levant.

\section{INTRODUCTION}

Au cours de la première moitié du $4^{e}$ millénaire avant J.-C., dans le Levant-Sud, apparaît une nouvelle catégorie morpho-technique de récipients céramiques. Ce sont des bols au profil en « $V$ » travaillés sur un instrument rotatif ${ }^{l}$. D'après la littérature archéologique ${ }^{2}$, ils représenteraient $40 \%$ à $60 \%$ des productions céramiques villageoises. Leur apparition est concomitante avec le développement de grands villages, la

1. BAIFIT, 1962 ; COMMIN(it:-PlillikIN, 1987. $199($ ).

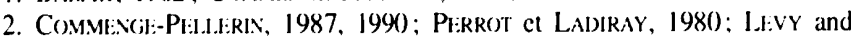
MINAHEM. 1987; USSISHKIN, 1980. mise en place de réseaux d'échanges à longue distance, une occupation différenciéc du territoire, des aires d'inhumation spécialisées, des lieux de culte ${ }^{3}$. Ils apparaissent, dès lors, comme des objets privilégiés pour tenter de comprendre quelques éléments de la dynamique des sociétés du $4^{\mathfrak{e}}$ millénaire, les faits techniques étant, par essence, étroitement imbriqués avec les systèmes socio-économiques ${ }^{4}$.

L'étude qui suit propose, dans cette perspective, une analyse technologique des bols en " $V$ " menée sur un ensemble archéologique chronologiquement bien circonscrit, à savoir

3. Plerrot et LADIRAY, 1980); L.FiY, 1985: Li:VY et Hol.I. 1988.

4. e.g. DOBRES and HoFWAN, 1994: LhMONNER. 1993. 
les céramiques du site d'Abu Hamid. Ce site, localisé dans la moyenne vallée du Jourdain, fut occupé de la fin du $6^{\mathrm{e}}$ millénaire au milieu du $4^{\mathrm{c}}$ millénaire avant J.-C. Les bols en forme de $« \mathrm{~V}$ » sont présents dans les couches datées de la première moitié du $4^{\mathrm{e}}$ millénaire avant J.-C. ${ }^{5}$.

Nous nous interrogerons tout d'abord sur le processus de fabrication de ces bols, sur les modalités d'exécution (qualité du travail, durée de fabrication, habiletés requises, instruments utilisés), sur les lieux de production et, enfin, sur les quantités produites. Ces informations permettront, ensuite, de faire des hypothèses sur la fonction des bols au profil en « $V$ », sur le statut socio-économique des potiers qui en sont les artisans, sur la nature des réseaux d'échanges qui s'y rapportent et, enfin, sur l'origine de cette nouvelle catégorie morphotechnique céramique.

\section{MÉTHODOLOGIE}

L'étude technologique céramique met en jeu deux niveaux d'observation complémentaires qui portent d'une part sur les macro-traces, d'autre part, sur les micro-fabriques. Leur compréhension implique l'élaboration préalable d'un référentiel interprétatif qui propose des traits diagnostics, dégagés comme tels suite à une analyse des mécanismes à l'ceuvre dans leur formation ${ }^{6}$. Les référentiels ici utilisés ont été élaborés afin de distinguer les différentes techniques de façonnage au tour ainsi que les différentes méthodes de façonnage afférentes à la technique de l'élaboration au tour ${ }^{7}$ (pour une définition de technique et méthode, voir infra: encadré).

L'étude des macro-traces et des micro-fabriques a été menée sur les céramiques des niveaux supérieurs d'Abu Hamid. Une série de tris successifs a permis rapidement d'organiser, selon des critères technologiques, une très grande quantité de tessons.

Le premier tri eut pour effet de distinguer, à partir de macro-traces significatives, les pièces élaborées au tour, des pièces façonnées sans l'aide de l'énergie cinétique rotative (abrégé ECR). Une pièce élaborée au tour se définit comme une pièce dont l'ébauche a été façonnée sans ECR mais dont la préforme, en revanche, a été mise en forme à l'aide de l'ECR (pour une définition de ces termes, voir infra). Les

5. Doll.Fis et KaFAF, 1988.

6. ColrTY and Rot:X, 1995.

7. RotX et CourTy, sous presse. Les expérimentations se sont déroulées au Centre Historique et Archéologique de Lejre (Danemark) en collaboration avec Inger Hildebrandt et Lizbeth Tvede-Jansen. pièces douteuses, c'est-à-dire les pièces présentant des traces de mouvement rotatif, mais non de façonnage à l'aide de l'ECR, étaient mises de côté momentanément jusqu'à leur identification technologique définitive à l'échelle microscopique (organisation caractéristique des domaines argileux en fonction des techniques et méthodes de façonnage). Les tessons appartenant au groupe des récipients élaborés au tour et dont la longueur était supéricure à $2,5 \mathrm{~cm}$ ont été décrits. Ceux dont la longueur était inférieure à $2,5 \mathrm{~cm}$ ont été simplement décomptés. Au total, 1276 tessons ${ }^{8}$ et 35 récipients entiers ont ćté identifiés comme appartenant au groupe des céramiques élaborées au tour. Ils comprennent 336 fragments de bases ou bases entières, 245 fragments de bords et 136 de panses de longueur supéricure à $2,5 \mathrm{~cm}, 359$ fragments de bords et 200 de panses de longueur inférieure à $2,5 \mathrm{~cm}$.

La technique de l'élaboration au tour peut être réalisée selon différentes méthodes, une méthode se définissant par l'agencement des opérations de façonnage. Un examen de l'ensemble des pièces élaborées au tour s'est révélé nécessaire pour reconstituer les méthodes. En effet, les macro-traces caractéristiques de façonnage sont le plus souvent oblitérées par les opérations de finition et il est illusoire de penser qu'un exemplaire de récipient entier pourrait, à lui seul, révéler l'ensemble du processus de fabrication. En revanche, l'examen de centaines de pièces permet de retrouver les quelques traits diagnostics qui vont permettre de reconstituer le puzzle, partant du principe que les pots d'un même type morphologique, appartenant à un assemblage homogène et présentant le même aspect morpho-technique, sont façonnés selon les mêmes méthodes et techniques.

Les récipients élaborés au tour ont été classés ensuite selon cinq catégories définies par l'épaisseur et la qualité de façonnage. Plus une céramique est fine, plus il est difficile de la façonner au tour en raison du danger de déformer des parois fines soumises à l'exercice de l'ECR. Les quatre premiers groupes ont été établis en fonction de la distribution des épaisseurs des panses mesurées à mi-parcours entre la base et le bord ou à $2,5 \mathrm{~cm}$ en-dessous du bord, sachant que les épaisseurs varient peu entre ces deux points de mesure : G1) de 0 à $3,5 \mathrm{~mm}, \mathrm{G} 2$ ) de 3,5 à $5 \mathrm{~mm}, \mathrm{G} 3$ ) de 5 à $7 \mathrm{~mm}, \mathrm{G} 4$ ) au-delà de $7 \mathrm{~mm}$. La cinquième catégorie regroupe les céramiques très épaisses dont le façonnage est peu soigné. Cette classification a pour but d'examiner la qualité des productions

8. N. vaillant fait un décompte de 1987 tessons de bols et jattes, $5(X)$ de plus que notre propre décompte. Cette différence est probablement liée au fait que $\mathrm{N}$. Vaillant a regroupé sous la dénomination bols et jattes des types morphologiques, alors que notre décompte de bols et de jattes s'applique à une catégoric morpho-technique, à savoir les récipients élaborés au tour. 
céramiques d'un point de vue synchronique et diachronique, ainsi que la pertinence de critères morpho-techniques pour déterminer des unités de production.

Méthode : enchaînement d'opérations pour réaliser une forme particulière. Cet enchaînement comprend des phases, des étapes et des opérations, chacune d'entre elles pouvant être réalisée selon des techniques différentes. On compte trois phases principales: le façonnage du corps (partie inférieure et supérieure), de l'ouverture (col et lèvre) et de la base. Le façonnage du corps peut être divisé en deux étapes: le façonnage de l'ébauche et de la préforme.

Ébauche : volume creux qui ne présente pas les caractéristiques géométriques finales du récipient. Une ébauche est obtenue par des opérations d'amincissement.

Préforme : récipient avec ses caractéristiques géométriques finales mais dont la surface n'a pas encore fait (ou ne fera pas) l'objet de traitement de finition. Une préforme est obtenue par des opérations de mise en forme.

Technique : modalités physiques selon lesquelles l'argile est façonnée. Ces modalités sont décrites sur la base de trois paramètres:

- la source d'énergie (pressions inter-digitales/palmaires vs pressions inter-digitales/palmaires combinées à l'énergie cinétique rotative);

- le type de pression (discontinues vs continues);

- la masse d'argile sur laquelle s'exercent les pressions (masse d'argile $v s$ éléments assemblés).

Parallèllement, une classification des micro-fabriques a été faite portant tout à la fois sur les récipients élaborés au tour et sur les récipients façonnés sans ECR. Cette classification a pris en compte les caractéristiques de la masse fine (type d'argile, abondance, pétrographie et tri granulométrique des inclusions fines), la pétrographie et la granulométrie des inclusions grossières. Les conditions de cuisson ont facilité l'identification, au microscope pétrographique, de la nature des matériaux utilisés. En effet, rares sont les céramiques qui présentent des traces de cuisson élevées ayant modifié les caractères minéralogiques de la fraction fine et entraîné une fonte partielle des inclusions grossières. La grande majorité des céramiques est caractérisée par un degré de cuisson moyen $\left(<700^{\circ} \mathrm{C}\right)$ qui $n^{\prime}$ a que faiblement modifié les propriétés de biréfringence des argiles d'origine et qui n'a pas entrainné d'altération thermique des inclusions grossières.
La classification des micro-fabriques, effectuée sous la loupe binoculaire, a porté sur trois ensembles céramiques:

1 - Une première classification générale a porté sur plusieurs centaines d'échantillons prélevés de manière aléatoire dans les différentes unités stratigraphiques de l'ensemble supérieur. Ce lot a fait l'objet d'un double tri simultané afin d'une part, de distinguer deux groupes: 1) les céramiques non élaborées au tour, 2) les céramiques élaborées au tour et, d'autre part, d'élaborer une classification de l'ensemble des types de pâtes. Ce tri a permis de reconnaître une variabilité considérable des types de pâtes du fait de la diversité des combinaisons entre trois attributs : (1) la minéralogie des fractions fines, (2) la pétrographie des inclusions grossières, (3) la granulométrie des inclusions grossières. L'identification de dix classes de fraction fine a permis de reconnaitre les types principaux qui rendent compte de la provenance des argiles. Au sein de chaque type, un nombre variable de sous-types (de 2 à 10) a été reconnu en fonction de la granulométric et de la pétrographie des inclusions grossières.

2 - Ensuite, un deuxième tri a été opéré sur plusieurs centaines d'échantillons de céramiques élaborées au tour (diagnostic basć sur les macro-traces) prélevés de manière aléatoire dans les différentes unités stratigraphiques de l'ensemble supérieur. Cettc étape a permis de créer une classification des micro-fabriques élaborées au tour et de la comparer à la classification générale.

3 - Un troisième tri a porté sur plusieurs centaines d'échantillons repérés par unité stratigraphique de l'ensemble supérieur et séparés en deux groupes sur la base de l'observation des macro-traces: (1) non élaborées au tour, (2) élaborées au tour. Cette étape a permis d'évaluer la variabilité diachronique des types de pâtes en fonction des différentes techniques de façonnage.

Ensuite, cette classification a ćté testée et affinée par l'étude au microscope pétrographique de 242 lames. Un à dix échantillons de céramiques ont été sélectionnés par types de pâte, auparavant identifiés à la loupe binoculaire en fonction de la variabilité de la texture de la fraction fine et des caractères des inclusions grossières. L'analyse au microscope pétrographique a permis d'une part, d'identifier la nature géologique des matériaux utilisés, d'autre part d'évaluer le degré de préparation des pâtes. 

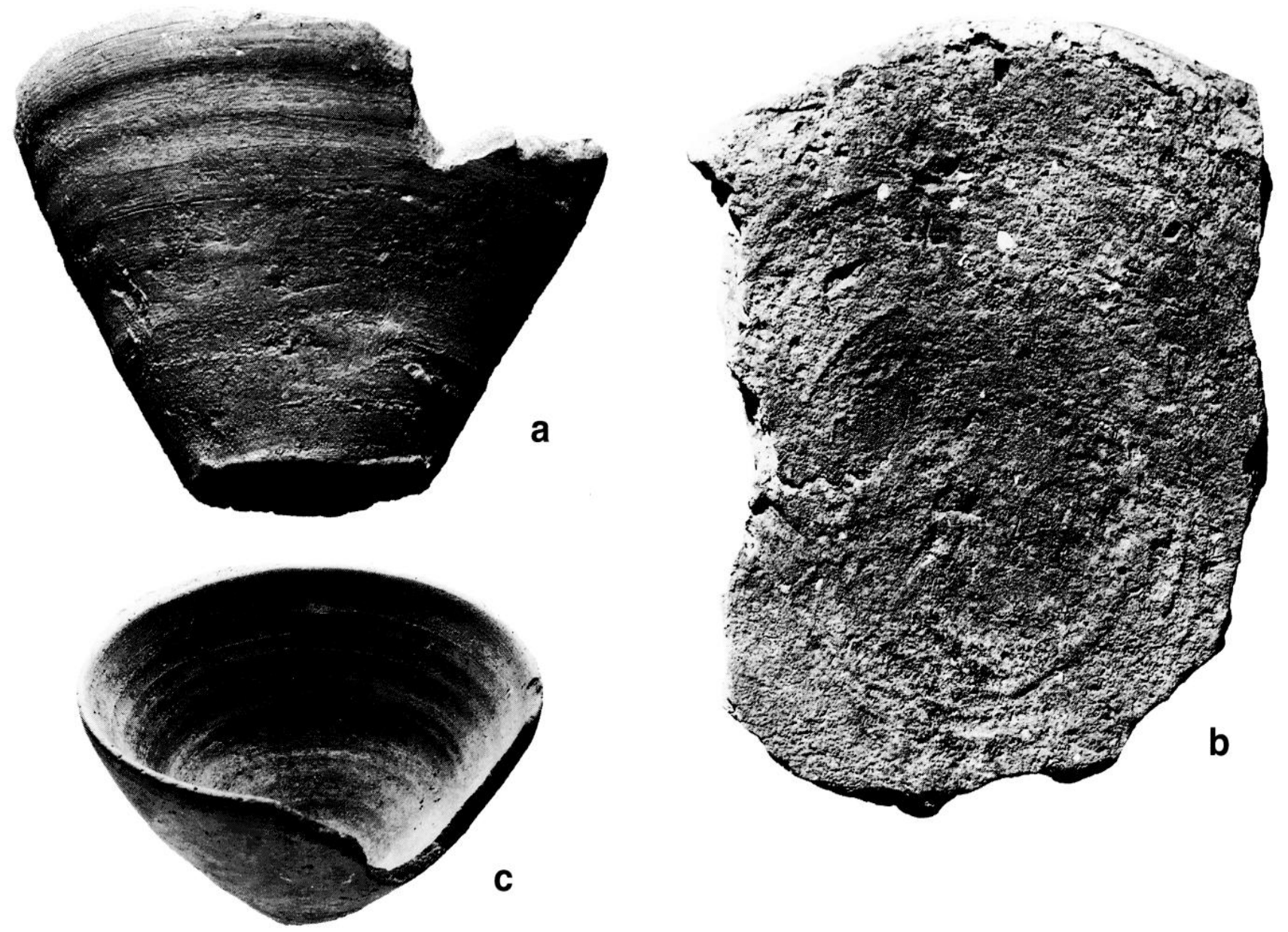

Planche 1: Bols au profil "en V»élaborés au tour. $a$ : Bol d'Abu Hamid, $b$ : fond d'un bol d'Abu Matar révélant les traces de colombin du support sur lequel reposait le bol. c, bol d'Abu Matar ( $b, c$, comm. J. Perrot).

\section{RÉSULTATS}

\section{MORPHOLOGIE DES RÉCIPIENTS ÉLABORÉS AU TOUR}

Les récipients élaborés au tour, selon le processus de façonnage décrit ci-après, sont pour l'essentiel des bols et des jattes au profil en «V» (Pl. I), ces deux types morphologiques ne se distinguant que par les dimensions de l'ouverture ${ }^{9}$. Ce résultat est comparable à ceux exposés dans la littérature archéologique où sont mentionnés essentiellement comme repris au tour ces deux types de récipients. L'utilisation de la technique de l'élaboration au tour pour les pots fermés est anecdotique. Elle a été observée à Abu Hamid sur les exemplaires suivants :

9. D'après la typologie de N. VAllLANT, à paraître. 10. In: Vall.LA.T, fig.10: $10,11,12$.
- un pot fermé à 6 oreillettes ( $\mathrm{n}^{0}$ de catalogue : 5569-1). La finition est remarquable et le processus de fabrication strictement le même que celui des bols et des jattes. C'est le seul pot à oreillettes rencontré qui ait été ainsi façonné, les autres pots entiers de ce type n'ayant pas été repris au tour ${ }^{10}$ (no 2368-1, 2132-1, 2139-1);

- un petit pot fermé à fond plat et à paroi convexe $\left(n^{0} 3242-4\right)$;

- un récipient fermé à lèvre redressée droite et bord aplati (no 2175-23);

- deux récipients fermés à lèvre éversée $\left(\mathrm{n}^{\circ}\right.$ 2425-19, 2439-16).

Précisons que la catégorie morphologique, bol et jatte, peut, en fait, recouvrir deux classes technologiques, certains bols et jattes n'ayant pas été élaborés au tour. Nous nous attacherons ici à la seule étude des récipients élaborés au tour. 

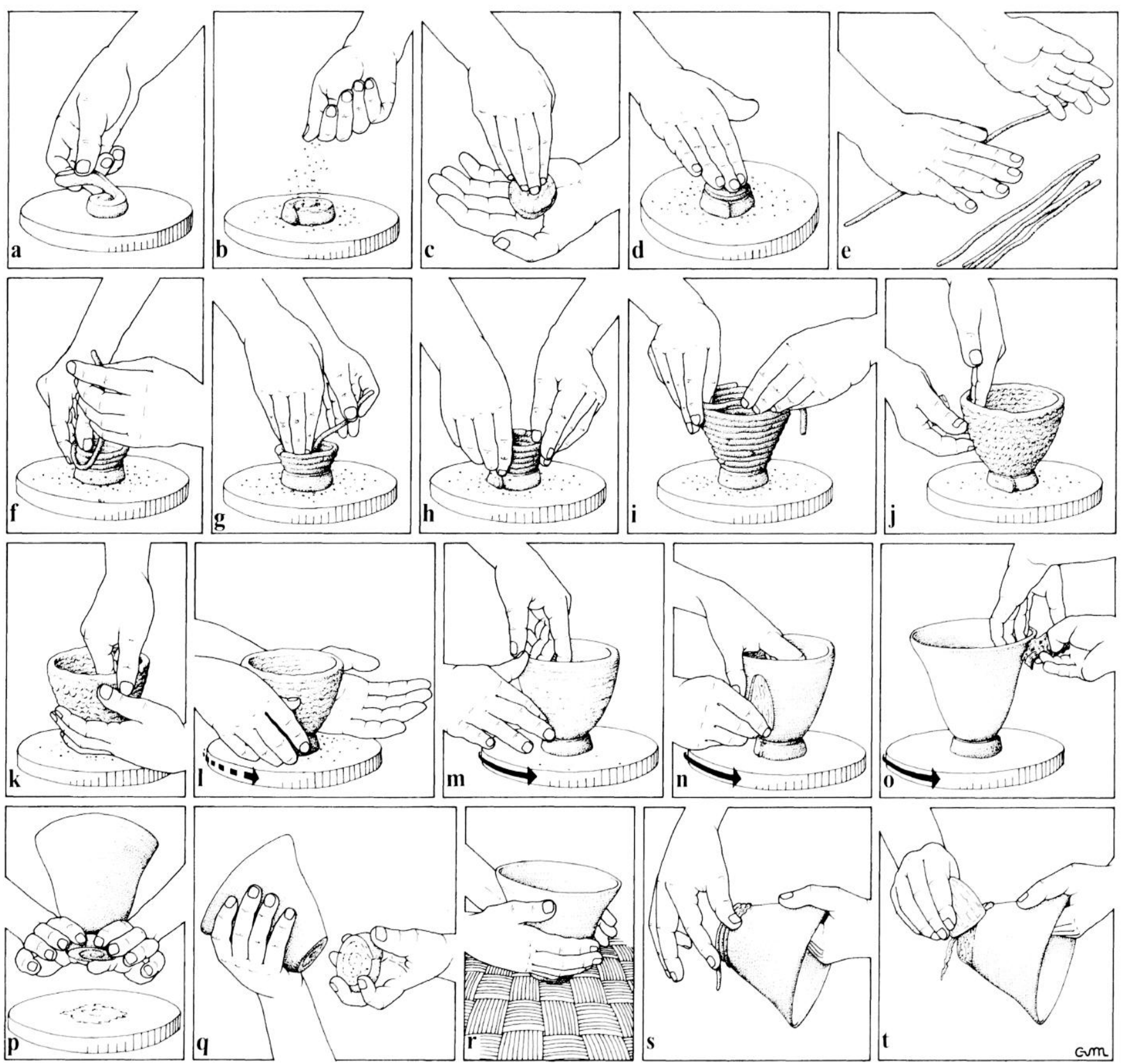

Fig. 1 : Chaine opératoire décrivant le façonnage des bols "en V" élaborés au tour: a: fixation d'un support en forme de colombin sur le tour, $b$ : "saupoudrage" de sable du support. $c:$ façonnage du fond, $d$ : pose du fond sur le support, e: façonnage des colombins, $f$ : montage de la base au colombin, g: renforcement de la base interne avec un on deux colombins, h-i: montage de l'ébauche au colombin. j-k : jointure des colombins par pressions discontinues, l: centrage du bol, m-n: amincissement par pressions comtinues à l'aide de l'ECR (énergie cinétique rotative), o: mise en forme par pressions continues à l'aide de l'ECR. p: enlèvement à force du tour, q: décollèment du support de la base, $r$ : séchage sur une natte en vamnerie, $s$ : rajout de colombins sur la face externe. $t$ : lissage avec une estèque en bois. 


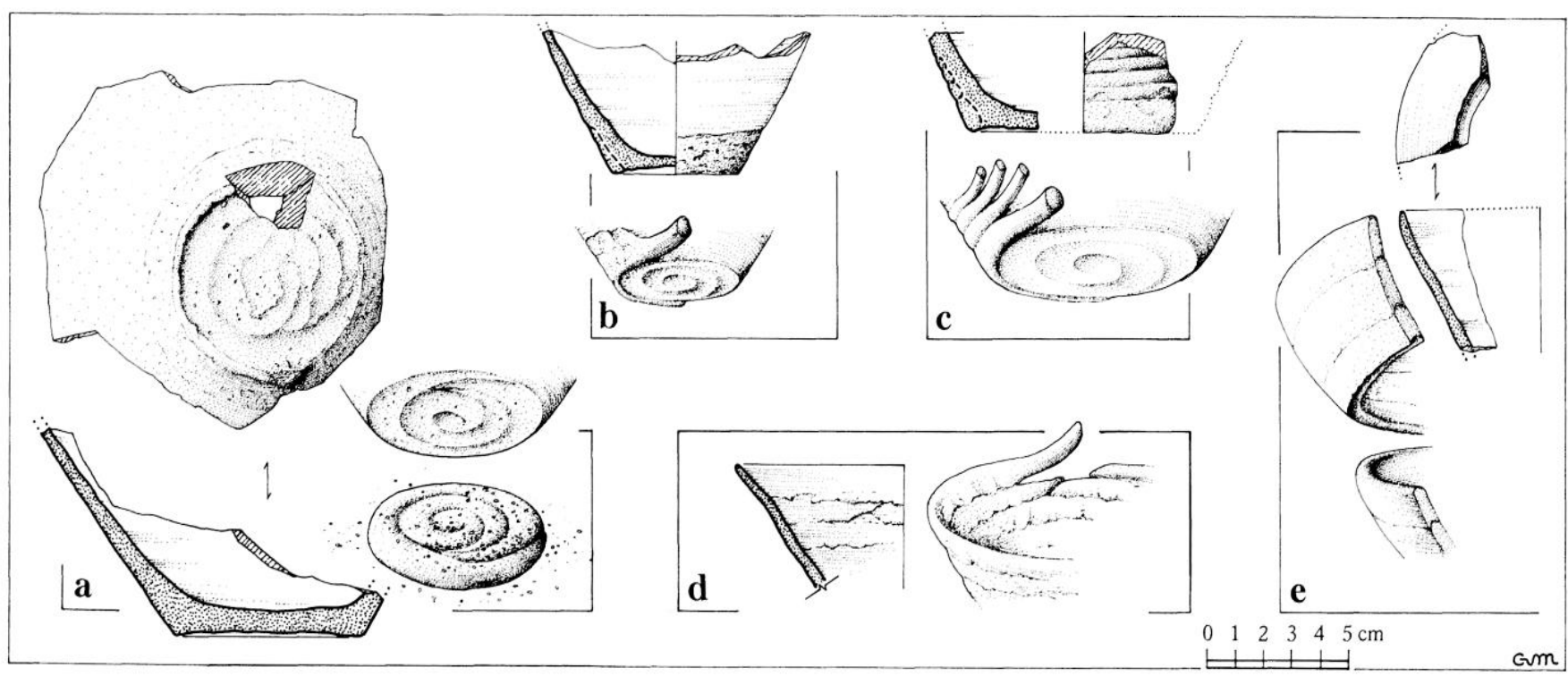

Fig. 2 : Traits diagnostics de façonnage. a : fond de bol présentant les traces du colombin ayant servi de support, b et $c:$ colombins placés sur la base externe alors que l'argile a atteint la consistance du cuir. Ces colombins sont ensuite aplanis à l'aide d'une estèque, d) jonction de colombins disposés en anneaux, e) fracture à la jonction de deux colombins.

\section{PROCESSUS DE FABRICATION DES RÉCIPIENTS ÉLABORÉS AU TOUR}

Après examen des pièces entières, il est apparu rapidement que les bols et jattes d'Abu Hamid n'étaient pas tournés sur motte, mais élaborés au tour. La chaîne opératoire de façonnage, ou processus de fabrication, est identifiable à partir de traits diagnostics (fig. 1 et 2). Elle est identique pour tous les bols et jattes qui présentent des traces de façonnage avec ECR (PI. I).

\section{FAÇONNAGE}

Le processus de façonnage comprend douze étapes distinctes:

1. Façonnage du fond à partir d'une petite masse d'argile amincie en galette (fig. $1 \mathrm{c}$ ).

Traits diagnostics: masse homogène. Pas de sillon ou de fracture suggérant des fonds fabriqués à partir de colombins.

2. Montage de la panse à l'aide de colombins disposés en anneaux (fig. 1 e-i). Le diamètre des colombins est compris entre $0,5 \mathrm{~cm}$ et $0,7 \mathrm{~cm}$.

Traits diagnostics: visibilité de jonctions de colombins à différentes hauteurs, y compris sur de petits bols, ce qui ne peut s'obtenir avec le montage d'un colombin en spirale. Les jonctions de colombins sont visibles sous forme de marques en forme de $\mathrm{X}$ que peut souligner une léger relief (fig. 2 d).
En outre, les bols présentent de nombreuses fractures caractéristiques de fractures de colombins observables également à différentes hauteurs de la panse (fig. 2 e).

3. Renforcement de la base par ajout d'un ou deux colombins sur la partie inférieure des parois internes (fig. $1 \mathrm{~g}$ ).

Traits diagnostics : la section des bases montre clairement un premier colombin qui assure la jonction entre le fonds et la partie inférieure de la panse, puis un second colombin, accolé à la face interne du premier. Ce dernier est destiné à renforcer la jonction entre la panse et le fonds qui sera fragilisée au moment de l'élaboration au tour. Celle-ci requiert une forte humidification de l'argile ce qui a pour conséquence une accumulation d'eau à la base. Cette eau a tendance à «couper» les parois et à fragiliser les points de discontinuité. Parfois, un troisième colombin est rajouté, à la jonction entre le second colombin et la panse afin toujours de renforcer la partie inférieure du récipient. Ces rajouts de colombins augmentent l'épaisseur des panses inférieures, comprise alors entre $1 \mathrm{~cm}$ et $2 \mathrm{~cm}$. En revanche, le fonds, qui n'est pas transformé, peut présenter une épaisseur de l'ordre de $3 \mathrm{~mm}$ seulement.

4. Jointure des colombins par pressions discontinues (fig. $1 \mathrm{j}-\mathrm{k}$ ).

Traits diagnostics : la jointure des colombins par pressions discontinues a pour effet de créer ce que l'on appelle des «ourlets» de jointure. Ces ourlets, comme leur nom l'indique, correspondent à des replis obtenus par étirement de l'argile 
d'un colombin sur l'autre afin de souder les colombins entre eux. Un ourlet de jointure est caractérisé par un tracé irrégulier légèrement en relief. Après mise en forme avec ECR, ce tracé disparaît soit totalement, soit partiellement. Il est alors visible sous forme de sillons irréguliers qui peuvent se réduire à un trait d'orientation oblique par rapport aux striations horizontales qu'engendre l'utilisation de l'ECR pour façonner l'argile.

5. Fixation de l'ébauche sur le tour. Le pot a pu être fixé sur le tour dès le façonnage du fond ou une fois l'ébauche terminée. Il est, en fait, plus aisé de monter un pot au colombin en le plaçant sur un objet rotatif qu'en le laissant sur une base fixe. Mais, quelle que soit l'étape de la chaîne opératoire à laquelle le pot était fixé sur le tour, le problème des modalités de fixation se pose en termes identiques. Il s'agit d'une part de fixer le pot de telle manière à ce qu'il résiste à la force centrifuge au moment du façonnage avec ECR, d'autre part de le fixer en fonction des modes de détachement possibles. Deux modes de fixation ont été observés.

a) Le pot est fixé sur le tour par le biais d'un support en argile en forme de gros colombin enroulé en spirale (figure 1 a-b). La face inférieure du support est fixćc au tour par scule adhésion d'une argile humide à un matériau «dur» (bois, argile séchée). Le maintien du support peut être renforcé par deux morceaux d'argile placés sur le côté, fixant ainsi le support au tour. La face supérieure du support est humidifiée, puis «saupoudrée» de sable afin de recevoir le fonds du pot qui a été ou sera monté au colombin avant d'être élaboré au tour. L'humidification du support permet une bonne adhésion du récipient au support, tandis que l'ajout de sable rend facile le détachement du pot, une fois le façonnage terminé. Le détachement consiste à décoller le support du fond, une fois celui-là enlevé, à force, du tour (fig. I p-q). Le décollement se fait d'autant facilement que le support présente une surface irrégulière et donc rend partielle l'adhésion entre le support et le fond du récipient. De ce point de vue, les supports formés d'un gros colombin sont plus adaptés à ce mode de détachement que les masses à surface plane.

b) Le pot est directement fixé au tour selon les mêmes principes que le support d’argile. Dans ce cas, le pot est détaché du tour à l'aide d'une fícelle. Ce cas a été observé pour deux exemplaires seulement.

Traits diagnostics: a) dans le cas du mode de fixation par l'intermédiaire d'un support en argile, les fonds présentent des surfaces externes irrégulières du point de vue tant de la texture que du plan. La texture est "grumeleuse», "bosselée», présentant des éléments grossiers piégés dans une argile fine.
Elle est caractéristique de l'empreinte argileuse de surfaces sableuses ou terreuses. Sur certains fonds, on peut distinguer, en relief, un très léger tracé en spirale qui témoigne d'une adhésion du fonds à un support en forme de colombin (fig. 1 a). Ce tracé peut totalement disparaître lorsque la surface du support en colombin est fortement aplanie au moment de sa fixation au tour à l'aide de l'ECR. Certains fonds sont plans, d'autres, en revanche, sont quasi convexes. La très grande variabilité morphologique des fonds externes vient argumenter l'hypothèse selon laquelle les fonds des pots ne reposaient pas directement sur les surfaces des tours, mais sur des supports non standards, souvent renouvelés.

b) Le mode de fixation direct sur le tour est déduit du mode d'enlèvement. Cclui-ci correspond à un détachement avec ficelle alors que le tour est activé ce dont témoignent des stries concentriques caractéristiques.

6. Centrage de l'ébauche (fig. 1 l). Cette opération est nécessaire pour travailler les parois d'argile à l'aide de l'ECR. Dans le cas d'une ébauche montée sans ECR, le centrage consiste à placer le pot de telle manière à ce qu'il tourne autour de l'axe central du tour. Ce placement se fait par à coups successifs sur le support ou la base du pot (selon le mode de fixation), tout en le faisant tourner. En aucun cas, il ne nécessite un montage préalable du pot sur le tour.

7. Amincissement de l'ébauche par pressions continues à l'aide de l'ECR (fig. $1 \mathrm{~m}-\mathrm{n}$ ). Lors de cette opération, les «ourlets» de jointure de colombins sont effacés et la morphologie des parois modifiées. Des pressions continues différenticlles répétées, exercées à l'aide de l'ECR au niveau de la jointure des colombins, favorisent l'effacement des ourlets et la jointure des colombins entre eux, transformant ainsi l'ébauche en un solide homogène. Ces pressions sont exercées avec les doigts ou avec des outils de type estèque en bois. Ces derniers facilitent la transformation des parois et l'effacement des ourlets de jointure.

Traits diagnostics : sillons parallèlles, ondulations concentriques sur faces interne et externe.

8. Mise en forme au tour du récipient à l'aide de l'ECR (fig. 1 o). La forme finale est donnée et les parois régularisées. A ce stade-là, le tour est activé plus lentement afin d'éviter des déformations de parois devenue fines et fragilisées par la quantité d'eau requise pour leur travail avec ECR.

Traits diagnostics: sillons parallèlles, parois rectilignes. Une rectilinéarité de paroi combinée à une absence de marque d'ourlets de colombins traduit la mise en forme soignée d'une ébauche dont l'amincissement fut achevé avant que les parois ne se saturent en eau. 
9. Enlèvement du récipient (fig. I p-q). a) Une fois le tour arrêté, le support, avec son récipient, est saisi par les mains et détaché du tour. Le support est ensuite décollé du fonds du récipient. b) Le pot est détaché à l'aide d'une ficelle pendant que le tour est activé.

Traits diagnostics : Voir les traits diagnostics décrits dans l'étape 5.

10. Le récipient est ensuite mis à sécher sur des nattes en vannerie (fig. $1 \mathrm{r}$ ).

Traits diagnostics: les fonds dont la surface est plane, c'est-à-dire les fonds qui ont pu imprimer le support sur lequel ils ont séché, présentent des traces de vannerie.

11. Renforcement de la base externe (fig. $1 \mathrm{~s}-\mathrm{t}$ ). Une fois que l'argile a atteint la consistance du cuir, le pot est repris à la main, une main en support à l'intérieur du pot. Des petits colombins ( 3 ou 4) sont rajoutés autour de la base externe du récipient et aplanis à l'aide d'une estèque en bois, sans l'aide de l'ECR. Le renforcement de cette base, sur une surface de 1,5 à $2 \mathrm{~cm}$, répond au problème technique que soulève l'utilisation de l'ECR pour façonner des ébauches montées à partir d'éléments assemblés et dont le fond présente de fortes discontinuités : la quantité d'eau utilisée fragilise la base non seulement lors du façonnage, mais également pendant l'opération de séchage. La rétraction d'eau sur les zones de discontinuité crée des fissures. Ces zones concernent, en l'occurrence, la jonction fond/paroi qui n'est jamais transformée en un solide réellement homogène, compte tenu probablement d'un fond trop mince au départ, et sur lequel ne peut «s'exercer» de véritables opérations d'amincissement unifiant fond et parois. La manipulation du récipient qui n'était pas encore sec devait être délicate, ce qui doit expliquer que la majeure partie des bols sont déformés comme l'exprime l'irrégularité des ouvertures.

Traits diagnostics : un bourrelet circulaire, plus ou moins irrégulier et épais, entourant les fonds, est nettement visible sur un grand nombre de bols. Il correspond au placement du premier colombin sur la base (fig. 2 b). Quelques bols montrent que deux à trois petits colombins ont été placés ensuite, appliqués successivement sur la panse inférieure. Ils sont visibles à l'œil nu dans les quelques cas où ils ont été mal aplanis (fig. $2 \mathrm{c}$ ). De manière générale, l'ajout de pâte que forment les colombins présente une texture différente de celle des parois façonnée à l'aide de l'ECR car la pâte a été travaillée à un degré d'hygrométrie différent (degré moindre que dans le cas du façonnage avec ECR). Cette texture est caractérisée par des sillons discontinus de morphologie variable, formés par l'entraînement des particules grossières de l'argile au moment de l'aplanissement des colombins avec un outil solide (pierre ou bois). Ces sillons peuvent être parallèles et concentriques. Toutefois, ils ne témoignent pas d'un façonnage avec ECR. D'une part, de tels sillons peuvent s'obtenir sans ECR compte tenu du parcours limité de l'outil sur l'argile. D'autre part, certaines pièces montrent des stries dont l'orientation oblique croisent des stries horizontales, ce qui correspond à la fin du parcours d'un outil manié sans ECR. Il est à noter que le renforcement de la base externe n'est pas toujours très soigné et que sa visibilité évidente, grossière parfois, est un marqueur technique caractéristique.

12. Le récipient est à nouveau mis à sécher sur une natte en vannerie.

Traits diagnostics: les fonds présentent des traces de vannerie qui «mordent» sur le bourrelet d'argile correspondant au renforcement postérieur de la base.

Une fois sec, le récipient peut être décoré. Une partie des bols et jattes présentent, sur les bords, des bandes rouges. La peinture est appliquée sans l'aide de l'ECR, probablement à l'aide d'un pinceau ou d'un tissu.

\section{DURÉE DE FABRICATION}

La chaîne opératoire de façonnage des petits bols en «V 》 d'Abu Hamid est complexe. Son exécution est longue. D'après nos expérimentations qui ont porté sur une soixantaine d'exemplaires ${ }^{11}$, il faut compter environ $40 \mathrm{mn}$ de façonnage pour un petit bol de $8 \mathrm{~cm}$ de haut et de $10 \mathrm{~cm}$ de diamètre : $30 \mathrm{mn}$ de montage au colombin et $10 \mathrm{mn}$ d'élaboration au tour. Le montage au colombin comprend la fabrication des colombins, leur pose et la jointure des colombins par pressions discontinues, autant d'opérations longues à mener lorsque les colombins sont montés en anneaux. L'élaboration au tour comprend les opérations d'amincissement et de mise en forme qui sont limitées dans le temps. En effet, ces opérations réclament une forte humification des parois. Celles-ci ne peuvent alors être travaillées au-delà d'un certain seuil, à moins d'encourir un risque d'affaissement général. Le renforcement de la base devait prendre environ $5 \mathrm{mn}$, ce qui élève à $45 \mathrm{mn}$ la durée du façonnage laquelle n'inclut pas les temps de séchage.

\section{INSTRUMENT ROTATIF}

De manière traditionnelle, on s'interroge souvent sur la dénomination des premiers tours : s'agissait-il de tournettes

11. ROL:X et COL'RTY, sous presse.

12. VAN DI:R LIIELW, 1994. 
ou de tours "véritables», de tours «rapides»? Cette question est liée à l'hypothèse selon laquelle les imperfections des premiers tours expliqueraient que les premiers récipients "tournés » étaient exclusivement de petites dimensions ${ }^{12}$. Car, très peu de tours ont été retrouvés, aucun à Abu Hamid. Toutefois, les caractéristiques techniques des bols et jattes ćlaborés au tour permettent de faire des hypothèses quant à l'instrument qui permit leur façonnage à l'aide de l'ECR.

Rappelons tout d'abord qu'un tour produit un mouvement rotatif qui n'est pas freiné au moment de son exploitation pour le façonnage de l'argile, au contraire de la tournette dont le mouvement rotatif est exploitable partiellement seulement (façonnage de la lèvre, lissage superficiel ${ }^{13}$ ). Le tour est nécessairement monté sur un axe alors que la tournette peut être un simple dispositif tournant.

Dès lors que bols et jattes ont leurs panses modifiées par l'ECR, on peut supposer l'existence d'un instrument rotatif dont lc mouvement n'a pas été freiné par l'application des pressions sur les parois au moment de leur amincissement et de lcur mise en forme. Les vitesses de rotation mises en jeu pour le tournage vont de 50 à $150 \mathrm{t} / \mathrm{mn}$, la vitesse étant d'autant réduite que le diamètre du récipient sur lequel s'exercent les pressions est grand ${ }^{14}$. "Ainsi, un col de quelques $\mathrm{cm}$ de diamètre est tourné à $150 \mathrm{t} / \mathrm{mn}$, tandis que les bords de grands plats de plusicurs décimètres de diamètre sont tournés à $50 \mathrm{t} / \mathrm{mn}$ ou moins. Ces vitesses de rotation, relativement aux diamètres concernés correspondent à des vitesses linéaires de 0,5 à $1 \mathrm{~m} / \mathrm{s}$ environ " 15 . De ce point de vuc, les petites dimensions des récipients élaborés au tour ne peuvent, en fait, argumenter en faveur d'un instrument dont les performances de vitesse ne permettraient pas le façonnage au tour de récipients de grandes dimensions. En effet, pour ces derniers, la vitesse du tour requise est alors inférieure à celle que requiert le façonnage de récipients de petites dimensions. En outre, il faut rappeler que, dans le corpus des céramiques élaborées au tour d'Abu Hamid, certaines jattes sont des pièces de grandes dimensions (ouv. $19 \mathrm{~cm}$, haut. $13 \mathrm{~cm}$ ).

On fera donc l'hypothèse que l'instrument rotatif utilisé pour le façonnage des récipients à l'aide de l'ECR était un tour à part entière, monté sur un axe, et dont la vitesse de rotation pouvait atteindre au moins $80 \mathrm{t} / \mathrm{mn}$ (vitesse obtenue lors de nos expérimentations pour façonner des bols de $8 \mathrm{~cm}$ de haut et de $10 \mathrm{~cm}$ de diamètre).
Reste la question de son fonctionnement. Pendant la phase de façonnage, était-il actionné en continu par un aide ou bien son volant d'inertie permettait-il une rotation autonome? La réponsc devrait être donnée, dans le futur, par des expérimentations appropriées sur des tours de potier analogues à ceux que l'on a rerouvés en contexte archéologique.

\section{HABILETÉS IMPLIQUÉES DANS L'ÉLABORATION AU TOUR}

Les habiletés impliquées dans l'élaboration au tour sont celles-là même que nous avons décrites pour le tournage ${ }^{16}$. Elles se définissent par une stabilité des avant-bras qui sont situés en asymétrie par rapport à l'axe de rotation du tour et par une associativité bimanuelle. Le contrôle moteur des deux mains se caractérise par l'exercice de pressions régulières et constantes, par une modulation de ces pressions en fonction de la plasticité de l'argile, de la vitesse du tour et de la nature de l'opération de façonnage.

Les habiletés impliquées dans le façonnage au tour sont particulièrement longues à acquérir en comparaison des habiletés développées dans la technique du colombin ${ }^{17}$. Contrairement aux secondes, ce sont des habiletés qui ne sont pas naturelles, mais spécifiques à la technique de l'élaboration au tour. Il faut compter plusieurs années pour être en mesure de les maîtriser totalement, c'est-à-dire en mesure de façonner au tour des récipients de toutes dimensions. Les données empiriques s'accordent sur une durée de dix ans. Récemment des études psychologiques ${ }^{18}$ ont démontré que dix ans d'apprentissage représentaient une «règle d'or» pour être expert dans une activité qu'elle soit d'ordre purement cognitive (le jeu d'échecs) ou motrice (les pratiques sportives).

En revanche, les habiletés impliquées dans la technique du colombin s'apparentent à des habiletés «naturelles» développées dès l'enfance pour des activités domestiques. Elles sont acquises rapidement, de l'ordre d'une à deux années.

Les habiletés impliquées dans le façonnage de l'argile contrastent ainsi fortement l'assemblage céramique d'Abu Hamid: d'un côté des récipients réclamant des habiletés spécialisées, les bols et les jattes élaborés au tour, de l'autre des récipients réclamant des habiletés moins spécialisées, les récipients façonnés sans l'aide de l'ECR.
13. ColkTY and Rolx. 1995: PII:RRI:T, 1995.
14. Rrt. 1981.
15. PII:RRLT, 1995: 35.
16. RorX et Corbitta, 1990; Gil.bert, sous presse.

17. Rol $x$ et Corbetta. 1990.

18. ERICSON and IJ:MNAN. 1996. 


\section{CARACTÉRISATION DES PÂTES UTILISÉES POUR LA PRODUCTION DES RÉCIPIENTS ÉLABORÉS AU TOUR}

\section{PERTINENCE DES CLASSIFICATIONS RÉALISÉES À LA LOUPE BINOCULAIRE}

Pour les céramiques élaborées au tour, l'étude au microscope pétrographique montre que la classification réaliséc à la loupe binoculaire est, dans l'ensemble, pertinente. Le taux d'erreur de diagnostic ne dépasse pas $10 \%$, et concerne surtout le pourcentage d'inclusions grossières. Il ne modifie pas significativement l'évaluation quantitative des différents types de pâte. La pertinence de cette classification tient à la faible variabilité des types de pâtes utilisées. En effet, pour les céramiques non élaborées au tour, l'étude au microscope pétrographique montre un taux d'erreur de près de $30 \%$ des diagnostics réalisés à la loupe binoculaire. Les erreurs sont liées, en grande partic, à la difficulté de discriminer une grande diversité d'assemblages pétrographiques d'inclusions grossières qui présentent des aspects semblables. Pour les différents types de pâtes fines, les erreurs sont liées à la difficulté d'évaluer correctement à la loupe binoculaire l'abondance et la nature des inclusions fines et la proportion de carbonates intimement mêlés aux argiles, marqueur important de l'origine des matériaux utilisés.

En conséquence, on retiendra qu'une classification pertinente d'un corpus de céramiques très diversifié implique, après un premier tri, une étape intermédiaire d'étude de lames minces au microscope pétrographique. Dans un deuxième temps, la liste des caractères diagnostics établic au microscope pétrographique permet de déterminer, à la loupe binoculaire, la représentativité des différentes classes de micro-fabriques. Dans le cadre de l'étude des céramiques d'Abu Hamid, cette seconde étape sera réalisée ultéricurement. Nous ne présentons ici qu'une reconnaissance des micro-fabriques des céramiques non élaborées au tour, suffisante pour dégager la spécificité des céramiques élaborées au tour.

\section{COMPARAISON DES MICRO-FABRIQUES DES CÉRAMIQUES ÉLABORÉES AU TOUR ET NON ÉLABORÉES AU TOUR (PI. II)}

L'assemblage des céramiques élaborées au tour est constitué en grande majorité $(90 \%)$ de pâtes fines caractérisées par une composante argileuse brun rouge à brun orangé, généralement carbonatée (abondance de fins limons calcitiques), finement mélangće à une composante limoneuse grossière
(40-80 $\mu \mathrm{m})$ constitués de grains de quartz anguleux à subanguleux $(40 \%)$ et de grains carbonatés $(60 \%)$.

A l'intérieur de ce groupe, la proportion de céramiques dépourvues d'inclusions grossières est de l'ordre de $80 \%$. Le sous-groupe à inclusions grossières montre un assemblage pétrographique homogène caractérisé par une composante de concrétions carbonatées millimétriques, identiques aux inclusions fines, par une composante de fragments de roches calcaires millimétriques, et plus rarement par une faible proportion de sables grossiers (500-200 $\mu \mathrm{m}$ ) constitué de quartz. arrondis et de silex anguleux.

Bien que ce groupe soit dans l'ensemble homogène, et aisément identifiable, le degré de carbonatation de la fraction fine, l'abondance et la morphologie des inclusions fines montre une nette variabilité entre les différentes lames minces étudiées. Cette variabilité ne permet pas, néanmoins, de reconnaître différents sous-types de ce groupe de pâte. Elle est plus vraisemblablement liéc à des nuances micro-régionales des matériaux d'origine.

Les caractères de la masse argileuse et des inclusions fines (esquilles de quartz typiques d'un transport éolien en suspension) montrent clairement que ce groupe de pâte provient des formations loessiques qui couvrent une grande partic du Ne$\mathrm{gev}^{19}$. Cette attribution est confirmée par la nature des inclusions grossières, constituées essenticllement de formations carbonatées identiques aux calcitisations pédologiques abondantes dans les sols et paléosols développés sur les loess du Negev ${ }^{20}$. La variabilité du degré de carbonatation des argiles, de la pétrographie et de la granulométrie des inclusions fines reflète les nuances spatiales et diachroniques des formations loessiques pédogénéisées du Negev ${ }^{21}$.

Ce groupe loessique n'est que faiblement représenté dans les céramiques non élaborées au tour $(<5 \%$ en première approximation). Il est généralement associé à des céramiques fines à moyennes (épaisseur $<1 \mathrm{~cm}$ ), et très rarement à des céramiques épaisses $(>1,5 \mathrm{~cm})$.

L'ensemble des $10 \%$ de céramiques élaborées au tour et non loessiques montre une diversité de pâtes fines qui peuvent ĉtre regroupées en dix types. Ces dix types sont tous présents dans l'ensemble des pâtes des céramiques non élaborées au tour. La différence essentielle entre les deux groupes techniques réside dans la faible proportion des sous-types à inclusions très grossières (taille de l'ordre de plusieurs millimètres) dans l'ensemble des céramiques élaborées au tour, alors que ces sous-types sont très largement représentés

\footnotetext{
19. Rorivon et al., 1987.

20. Ibid.

21. YAALON and DAX, 1974.
} 
Tableau 1 : Description sỵnthétique des caractères des pâtes céramiques élaborées au tour.

\begin{tabular}{|c|c|c|c|c|c|}
\hline Groupe & Masse fine & Inclusions grossières & $\% / \mathrm{T}$ & $\% / \mathrm{NT}$ & Provenance \\
\hline Groupe I & $\begin{array}{l}\text { Argile carbonatée brun orangé à } \\
\text { composante loessique }\end{array}$ & $\begin{array}{l}\text { Sables fins à moyens, calcaires, } \\
\text { subarrondis }\end{array}$ & $90 \%$ & $<5 \%$ & Negev \\
\hline Groupe II & $\begin{array}{l}\text { Argile micritique brun orangé à } \\
\text { micro-bioclastes }\end{array}$ & $\begin{array}{l}\text { Sables subarrondis, moyens à } \\
\text { grossiers, calcaires bioclastiques }\end{array}$ & $<5 \%$ & $20 \%$ & $\begin{array}{l}\text { Bassins latéraux de la moyenne } \\
\text { vallée du Jourdain }\end{array}$ \\
\hline Groupe III & $\begin{array}{l}\text { Argile carbonatée brun orangé à } \\
\text { limons sableux (quartz, } \\
\text { altérables, carbonates) }\end{array}$ & $\begin{array}{l}\text { Sables fins à grossiers (1) silex } \\
\text { anguleux, (2) basaltes arrondis, } \\
\text { (3) roches calcaires }\end{array}$ & $<5 \%$ & $20 \%$ & $\begin{array}{l}\text { Partie centrale de la moyenne } \\
\text { vallée du Jourdain (Abu Hamid) }\end{array}$ \\
\hline Groupe IV & $\begin{array}{l}\text { Argile carbonatée très fine brun } \\
\text { jaune }\end{array}$ & $\begin{array}{l}\text { Sables subarrondis, moyens à } \\
\text { grossiers de calcaires } \\
\text { bioclastiques ferruginisés, } \\
\text { d'ooïdes ferrugineux et de } \\
\text { roches siliceuses anguleuses }\end{array}$ & $5 \%$ & $20 \%$ & $\begin{array}{l}\text { Bassins latéraux de la moyenne } \\
\text { vallée du Jourdain (Pella?) }\end{array}$ \\
\hline Groupe V & Argile fine brune & $\begin{array}{l}\text { Sables arrondis, fins à moyens, } \\
\text { calcaires, basalte et silex }\end{array}$ & $<1 \%$ & $5 \%$ & $\begin{array}{l}\text { Haute vallée du Jourdain (sud } \\
\text { du Golan) }\end{array}$ \\
\hline Groupe VI & $\begin{array}{l}\text { Argile fine, brun rouge, peu } \\
\text { carbonatée }\end{array}$ & $\begin{array}{l}\text { Sables moyens, calcaires } \\
\text { arrondis et quartz anguleux }\end{array}$ & $<1 \%$ & $10 \%$ & $\begin{array}{l}\text { Epandages colluviaux à } \\
\text { proximité de la vallée du } \\
\text { Jourdain (Abu Hamid) }\end{array}$ \\
\hline Groupe VII & $\begin{array}{l}\text { Argile carbonatée, très fine, } \\
\text { beige }\end{array}$ & $\begin{array}{l}\text { Fragments anguleux de bivalves, } \\
\text { taille des sables moyens à } \\
\text { grossiers, anguleux }\end{array}$ & $<1 \%$ & $15 \%$ & Indéterminée \\
\hline Groupe VIII & $\begin{array}{l}\text { Argile fortement carbonatée, très } \\
\text { fine, brun jaune }\end{array}$ & $\begin{array}{l}\text { Sables subarrondis, moyens à } \\
\text { grossiers de calcaires } \\
\text { bioclastiques et dolomitiques }\end{array}$ & $<5 \%$ & $15 \%$ & $\begin{array}{l}\text { Bassins latéraux de la moyenne } \\
\text { vallée du Jourdain }\end{array}$ \\
\hline Groupe IX & Limons argileux carbonatés gris & $\begin{array}{l}\text { Sables fins, subarrondis, quartz } \\
\text { et carbonates }\end{array}$ & $<1 \%$ & $5 \%$ & Formations du Lisan \\
\hline
\end{tabular}

dans le groupe des céramiques non élaborées au tour. Les caractères de la masse fine et des inclusions grossières permettent d'avancer des hypothèses quant à la provenance de la plupart de ces pâtes (Tableau 1). Elles proviendraient essentiellement de la vallée du Jourdain avec, cependant, une faible proportion des pâtes d'origine locale. Celles-ci sont largement micux représentées dans l'ensemble des céramiques non élaborées au tour. Toutefois, elles ne constituent pas un assemblage dominant.

Enfin, signalons que, parmi les types de pâtes identifiés dans l'ensemble des céramiques non élaborées au tour, trois types, au moins, sont absents de l'ensemble du groupe des céramiques élaborées au tour :

- groupe NTA (10-20\%): argile brune organique, non carbonatée, à sables arrondis, quartzeux, moyens et bien triés ou à dégraissant organique (excréments animaux) ou à chamotte; la provenance de ce type de pâte est à rechercher à proximité de la Mer Morte, comme le confirme sa forte fréquence sur le site de Ghassoul;
- groupe NTB $(<5 \%)$ : argile carbonatée, brun rouge, à fragments calcaires grossiers anguleux, bien triés;

- groupe NTC $(<5 \%)$ : argile fine micritique brun jaune, à fragments calcaires grossiers anguleux, bien triés.

Les indices sont insuffisants pour déterminer la provenance des types NTB et NTC qui reflètent un choix de pâtes et de constituants grossiers singuliers, mais non spécifiques d'un contexte géologique.

\section{PRÉPARATION DES PÂTES}

L'analyse au microscope pétrographique permet de reconnaître deux qualités de préparation pour des pâtes fines identiques, en prenant en compte la distribution texturale des inclusions grossières et le degré de dissociation des domaines argileux, significatif des transformations hydriques et mécaniques subies par le matériau après son extraction. 

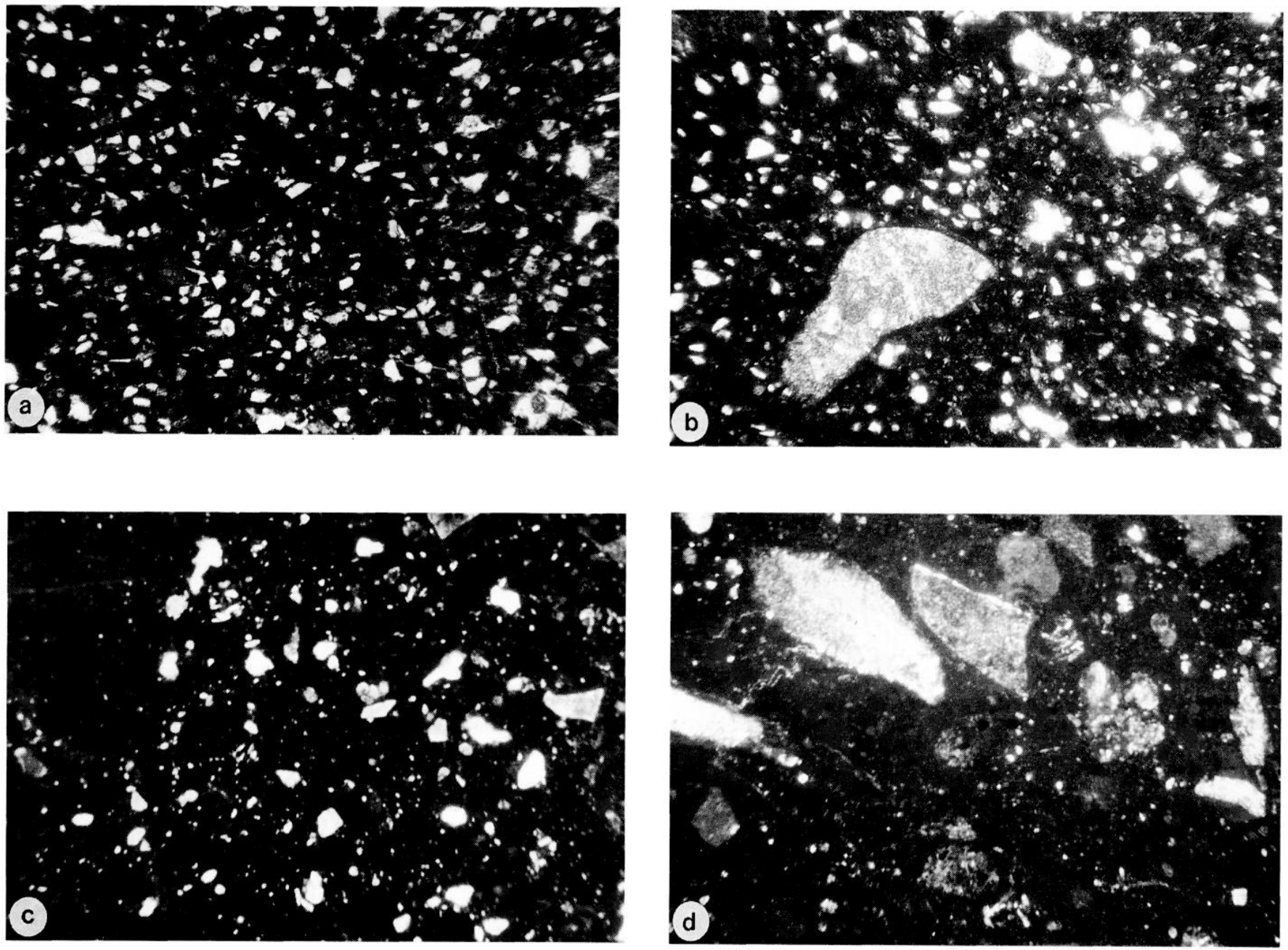

Planche 2: Exemple des différents groupes de pâtes des céramiques élaborées au tour: a: (iroupe I. pâte loéssique du Negè: bien préparée sans dégraissant grossier; sous-type pâte grossière $(L N)$. b: Groupe l, pâte loessique du Negè; mosenne'ment préparée, à inclusions de roches calcaires; sous-type pâte fine (LN). c: Groupe II. limons sableux rouges collariaux, mal triés, mal préparés; la faible carbonatation de la masse fine, la forte proportion de sables mal triés et la présence de fragments de crôites sédimentaires indiquent des matérianx pris dans des horizons de surface $(A),(L N)$. d: Groupe III, formation colluviale rouge de la movenne vallée du Jourdain mélangée de sables grossiers (silex, roches calcaires, grains de basalte); la forte carbonatation de la masse fine et la faible proportion de sables fins indiquent des matériaux pris dans des horizons B de sols (LP). e: Groupe IX, pâte fine carbonatée pronenant des dépôts lacustres du Lisan (LN). $f:$ Groupe V, argile fine brune provenant de la haute vallée du Jourdain, mélangée de sables alluriaux bien ralibrés èt de fragments millimétriques de roches calcaires $(L N)$. g: Groupe VII, argile carbonatée très fince beige mélangée de sables grossiérs provénant du concassage de coquilles de bivalves (LN). h: Groupe VIII, argile carbonatée très fine jaune orangé, mélangée de sables grossiers anguleux provenant du concassage de calcaires bioclastiques et dolomitiques (LP).

(a) - des pâtes soigneusement préparées : elles représentent la très grande majorité de l'ensemble de la production céramique.

L'identification d'une préparation des argiles par décantation s'appuie sur deux critères: (1) la présence d'un réseau régulier de fines figures de biréfringence dans les zones de contact des domaines argileux qui témoigne de la dispersion partielle du matériau, suffisante pour rompre les liens interagrégats acquis au cours du développement pédologique;
(2) la rupture granulométricjue nette au-delà des sables fins qui atteste l'élimination intentionnelle des inclusions supéricures à $100 \mu \mathrm{m}$. Cette distribution granulométrique n’est qu'exceptionnellement rencontrée dans des matériaux naturels, le plus souvent des loess fïns très bien triés, faiblement pédogénisés, absents de la région d'étude. L’écrasement des domaines argileux et leur organisation régulière indiquent que l'argile, une fois récupérée. a fait l'objet d’un malaxage soigné. 

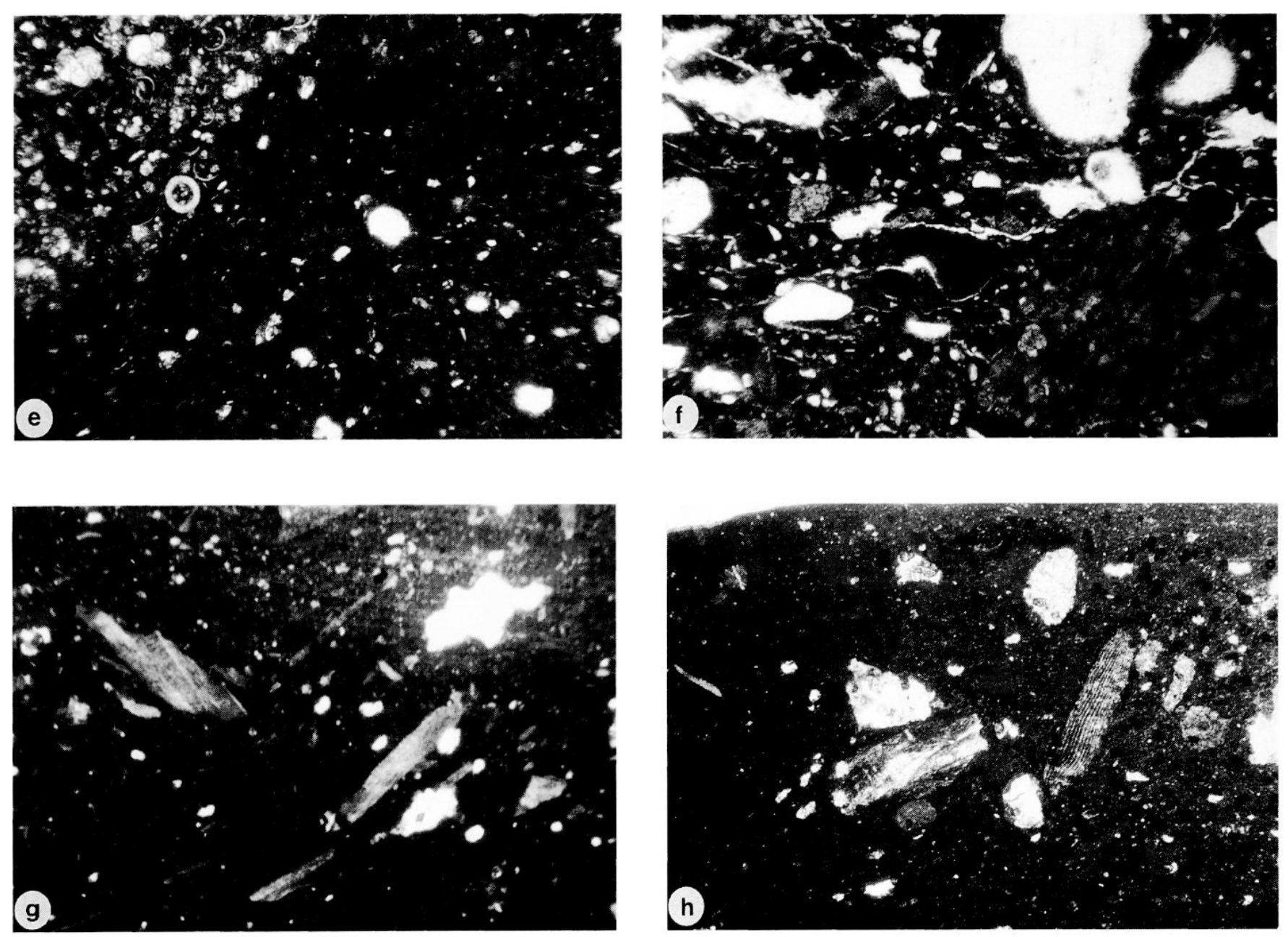

La réincorporation d’inclusions grossières a pu être identifiée pour certains types de pâtes, à l'exception du groupe I (loessique/Negev). Elle est marquée soit par la rupture granulométrique entre la pâte fine et les grains grossiers, soit par une anomalie entre l'origine de la pâte fine et la pétrographic des inclusions grossières, ce type d'association ne pouvant provenir de matériaux naturels. Il s’agit d’inclusions grossières calibrées pour lesquelles la granulométrie et la composition pétrographique permettent d'identifier deux origines :

- des inclusions grossières provenant de différentes strates de formations alluviales, telles celles de la vallée du Jourdain et des affluents latéraux (ex. groupe V, PI. II f);

- des inclusions grossières bien calibrées produites par concassage des matériaux, comme en témoigne leur morphologie anguleuse, surtout des roches calcaires et des coquilles. puis la sélection intentionnelle d'une classe granulométrique; cette production intentionnelle est limitéc aux groupes de pâtes VII et VIII (ex. planche II, get h) qui sont faiblement représentés dans les deux ensembles techniques (Tableau 1).

L'introduction d'inclusions grossières, dont le calibrage résulte de processus naturels, explique bien la variabilité des pâtes au sein d'un même groupe. Utilisant toujours le même type d'argile, les différentes unités de production ont tiré parti de la diversité des situations géologiques (formations sableuses lenticulaires ou à stratifications entrecroisées) pour collecter les matériaux de leur choix sans les modifier.

(b) - Des pâtes faiblement préparées: rares pour les céramiques élaborées au tour $(<1 \%)$ et faiblement représentées pour les autres $(10 \%)$.

Elles sont caractérisées par un continuum entre la masse argileuse, les inclusions fines et les inclusions grossières. Généralement, elles ne comportent pas d’ajout de constituants grossiers calibrés. De plus, les reliques de micro-agrégats argileux d'origine pédologique sont encore visibles en lames minces. Après extraction, les matériaux ont été humidifiés mais n’ont pas fait l'objet de décantation. 


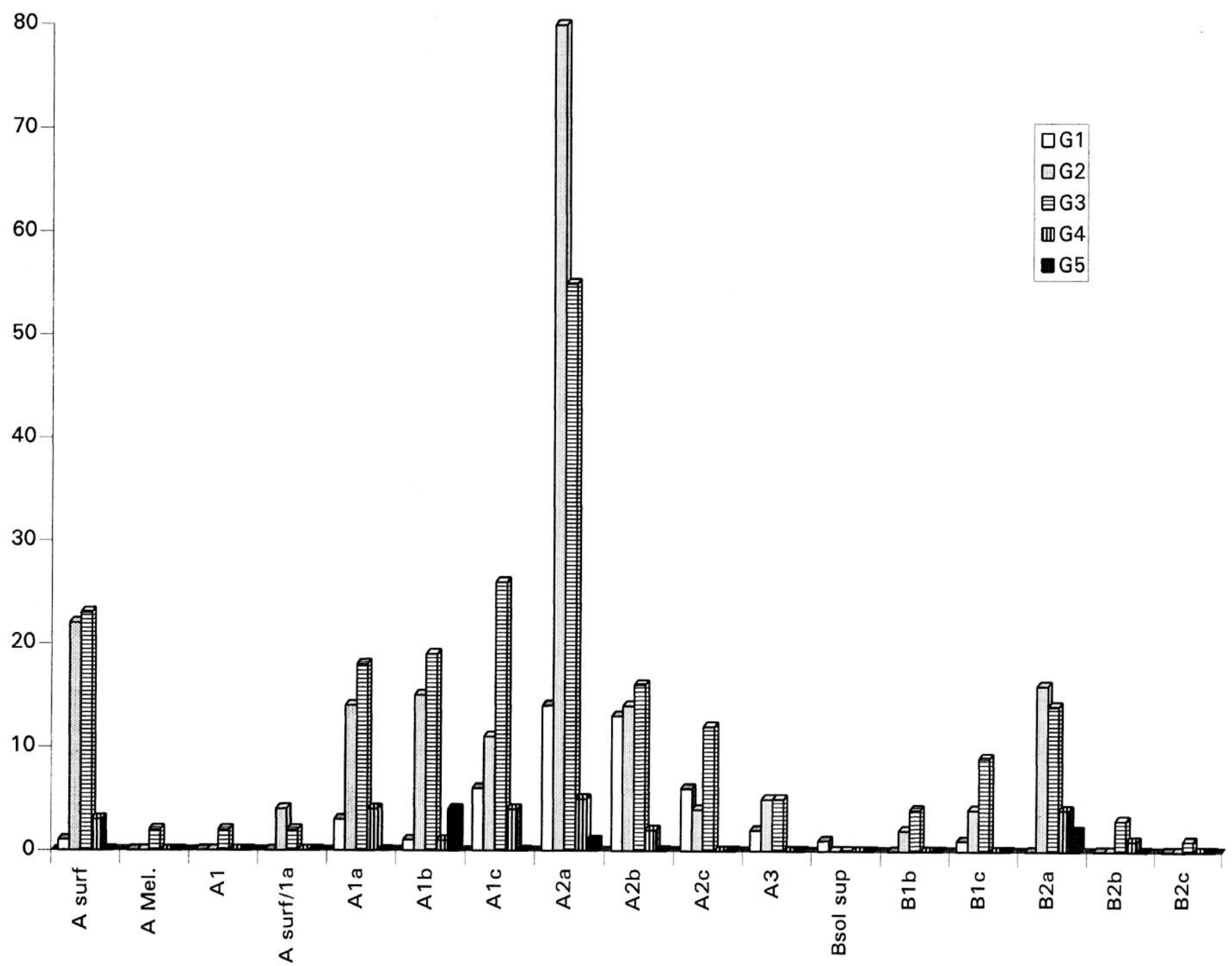

Fig. 3 : Répartition des récipients élaborés au tour par groupe qualitatif que définissent l'épaisseur des panses et la qualité de façonnage $(G I=$ de 0 à $3,5 \mathrm{~mm}, G 2=$ de 3,5 à $5 \mathrm{~mm}, \mathrm{G} 3=$ de 5 à $7 \mathrm{~mm}, \mathrm{G} 4=$ au-delà de $7 \mathrm{~mm}$. G5 regroupe les céramiques très épaisses dont le façonnage est peu soigné).

\section{VARIABILITÉ DIACHRONIQUE}

Au sein du groupe des céramiques élaborées au tour, la proportion des différents types de pâtes ne présente pas de variations significatives entre les différentes unités stratigraphiques de l'ensemble supérieur.

\section{QUALITÉ DE FAÇONNAGE ET QUALITÉ DE PRÉPARATION DES PÂTES}

Les cinq groupes que définissent l'épaisseur des panses et la qualité de façonnage sont quantitativement distincts (fig. 3). Les groupes 1, 4 et 5 sont faiblement représentés, au contraire des groupes 2 et 3 qui forment la très grande majorité de l'ensemble de la production $(85 \%)$. De ce point de vue, les groupes grossièrement façonnés sont anecdotiques au regard de la production globale.

De manière générale, si les cinq groupes sont présents dans les différentes unités stratigraphiques de l'ensemble supérieur, on observe pour tous, néanmoins, une forte concentration de la production pendant la phase $\mathrm{A} 2 \mathrm{a}$. Loin derrière, viennent les phases $A 2 b$ et Alc, Alb, Ala (fig. 4). 


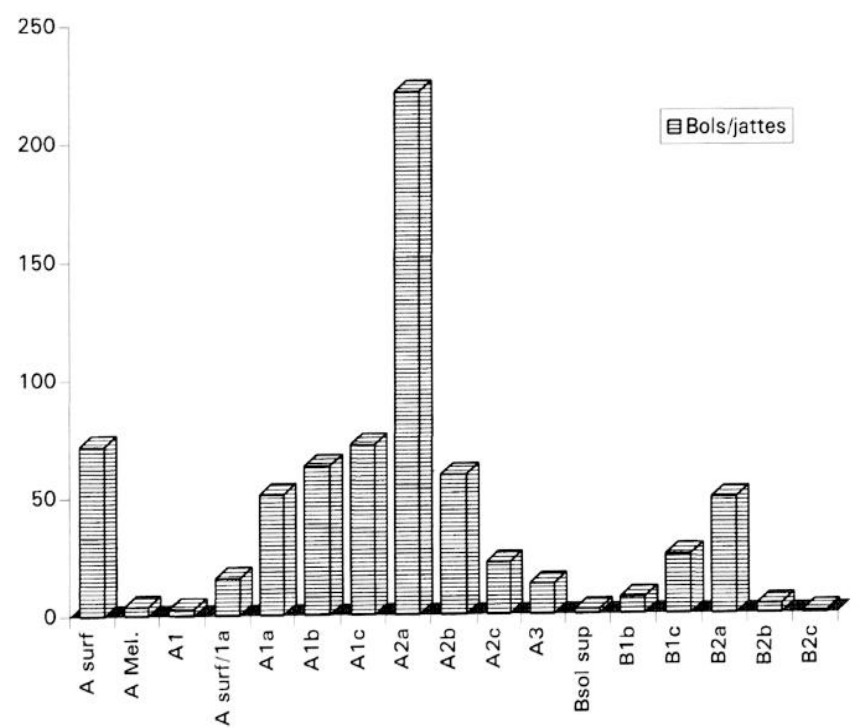

Fig. 4 : Répartition des récipients élaborés au tour par unité stratigraphique.

\section{QUANTIFICATION DE LA PRODUCTION DES RÉCIPIENTS ÉLABORÉS AU TOUR}

Ainsi que nous l'avons vu précédemment, le façonnage des bols élaborés au tour prend environ 45 minutes. En théorie, un potier peut donc en fabriquer 10 par jour (à raison d'une journée de travail équivalente à 7 heures $30 \mathrm{mn}$ ). S'il travaille l'équivalent de 3 mois par an (100 jours), sa production annuelle s'élèvera à 1000 pots environ. En admettant qu'un potier produise pendant 25 ans, sa production, à l'échelle d'une vie, sera de 25000 récipients. Cette même production prise sur 100 ans s'élèvera à 100000 récipients qui, une fois fragmentés, donneront jusqu'à un million de tessons. Cet exercice quantitatif, simple, est destiné à démontrer qu'un artisanat spécialisé fournit rapidement un nombre élevé de pièces ${ }^{22}$ et qu'il faut donc être prudent avant d'interpréter un nombre intuitivement élevé de pots en termes de «production intensive».

Qu'en est-il alors du nombre de bols et jattes élaborés au tour retrouvés sur le site d'Abu Hamid? On a retrouvé, au total, dans la zone fouillée 1276 tessons et 35 récipients entiers que l'on a pu identifier comme appartenant au groupe des céramiques élaborées au tour.

Prenons une hypothèse forte (nombre maximum de récipients) : à chaque base, et à chaque fragment de bord et de panse, correspond un individu, ce qui totalisent, avec les pièces entières, environ 1300 récipients élaborés au tour.

22. Rolx el Matakasso, sous presse.
Prenons, ensuite, une seconde hypothèse forte : la quantité de bols/jattes retrouvés dans le secteur dégagé est présente dans les mêmes proportions sur l'ensemble du site. Les bols ont été retrouvés sur une zone qui correspond à $5 \%$ de la surface totale de la partie du site où est implanté le chantier $\left(1200 \mathrm{~m}^{2}\right.$ fouillés sur une surface de 2,5 hectares). Les 1300 bols représenteraient alors $5 \%$ de la quantité de bols globalement utilisés en cette partie de l'agglomération, ce qui monterait le nombre de bols/jattes à 26000 . Si l'on divise maintenant ce chiffre par le nombre d'années correspondant à la durée d'occupation des couches renfermant les céramiques élaborées au tour, entre 150 et 200 ans, le nombre de bols «consommés» annuellement à l'échelle de ce quartier s'élèverait à $130 \ldots$ Cette petite production est réalisable en treize jours par un scul potier.

Un si faible taux de production laisse supposer que des particularités techniques comme l'enlèvement du tour à la ficelle ont pu être l'apanage d'un seul potier dont la production fut distribuéc sur une grande région (la trace de l'enlèvement à la ficelle n'est présente que sur deux exemplaires à Abu Hamid). Elle pourrait avoir été inventée par un potier qui se l'appropria en tant que marque spécifique de sa production.

D’après N. Vaillant (à paraître), les bols et jattes élaborés au tour représentent environ $60 \%$ de la production céramique du site d'Abu Hamid. Ce chiffre, retrouvé dans des ordres de grandeur approximatifs sur d'autres sites (entre $40 \%$ et $50 \%$ à Abu Matar, à Safadi, à Shiqmim ${ }^{23}$ ), a été calculé à partir du nombre de tessons de bols ou de jattes retrouvés. Un tel pourcentage, dont l'homogénéité d'un site à l'autre transgresse les problèmes de fragmentation différentielle d'un assemblage à l'autre ${ }^{24}$, pourrait être lié à un phénomène de sur-représentation propre aux vaisselles de petite taille. En effet, des études ethnoarchéologiques ont montré que les récipients de petite taille avaient une durée de vie plus courte que les gros récipients (jarres) ${ }^{25}$ et qu'il en résultait un biais à l'échelle d'une couche archéologique, les petits pots à durée de vie courte étant dès lors sur-représentés, dans une vision synchronique, par rapport aux vaisselles aux durées de vie plus longues. En outre, il ne faut pas sous-estimer le fait qu'au moment du tri des tessons, les fragments de panse des bols sont attribuables dès l'abord à un type (les bols en « $\mathrm{V} »$ ) étant donné, en particulier, l'aspect morpho-technique caractéristique des parois. En conséquence, ils sont susceptibles, a priori, de faire l'objet d'un échantillonnage beaucoup plus

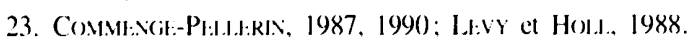

24. ORToN et al. 1993.

25. MAYOR, 1994. 
large que les panses des récipients à l'attribution typo-morphologique douteuse. Enfin, tous les bols et jattes sont généralement décomptés globalement alors que ces types morphologiques recouvrent deux classes technologiques distinctes. Il s'ensuit là-aussi une sur-représentation, dans certains cas, du nombre de récipients considérés appartenir au type bol/jatte repris au tour.

\section{INTERPRÉTATION ET DISCUSSION}

\section{VALEUR DES BOLS/JATTES ÉLABORÉS AU TOUR}

D'après les descriptions et représentations publiées dans la littérature archéologique, la très grande majorité des bols en « $V$ » des sites du Levant Sud (sites de la vallée du Jourdain, du Negev et des plaines côtières) ont été élaborés au tour selon le processus de façonnage décrit pour les bols d'Abu Hamid. Ce processus technique, de par les habiletés impliquées, peut être qualifié de «haute technicité». Il se distingue des processus de façonnage qui n'utilisent pas l'ECR et dont les habiletés impliquées sont moins complexes. Cette haute technicité, accrue par des préparations de pâte de grande qualité, fut réservée à un nombre limité de catégories morphologiques, les bols et les jattes. En effet, elle ne fut pas transférée à l'ensemble des récipients céramiques alors même que d'un point de vue technique, stricto sensu, aucune contrainte ne l'empêchait, que ce soit du point de vue de l'instrument utilisé ou des habiletés développées.

Objets donc exceptionnels, les bols et jattes élaborés au tour ont été retrouvés non seulement en contexte d'habitat comme à Abu Hamid, mais également en contexte funéraire ou cultuel, que ce soit dans les tombes à ossuaires de la région côtière où les bols en « $V$ » représentent $50 \%$ du mobilier céramique «d'accompagnement ${ }^{26}$, dans la nécropole de Shiqmim où «il semble que chaque individu ait été enterré avec au moins un bol à profil en « $\mathrm{V} »{ }^{27}$, ou encore au sanctuaire de En-Gedi ${ }^{28}$. Dans ces contextes (Azor, Ben Shemen, En-Gedi), les bols en « $V$ » sont accompagnés de bassins, de coupes à pied fenestré, de cornets, de grands vases à bords rentrants, de vases globulaires à oreillettes et de vases fusiformes, autant de récipients aux formes spécifiques qui se distinguent, non seulement par leur forme, mais également par leur facture, de l'ensemble des formes retrouvées dans

26. Perrot et Ladiray, 1980.

27. LeVY et Holl, 1988: 296.

28. USSISHKIN, 1980 . les différents faciès céramiques des sites d'habitat de cette époque.

Cet ensemble d'éléments technologiques et contextuels suggèrent alors que les bols/jattes en « $V$ », objets uniques de haute technicité, étaient réservés à des fonctions spécifiques qui ne se rattachaient pas à la sphère domestique/utilitaire. En effet, d'une part, on est curieusement dans une situation où une pratique technique est limitée, dans le temps et l'espace, au façonnage d'un seul type de récipient qui ne pose aucune spécificité de fabrication au regard des différentes techniques de façonnage connues. Une telle spécialisation s'inscrit hors des schémas classiques de division sexuelle du travail ou de spécialisation socio-professionnelle, qui peuvent envisager des pratiques techniques différentes pour des objets de même fonction et/ou statut. D'autre part, les exemples ethnographiques sont nombreux qui démontrent l'importance de l'association technique/fonction de l'objet pour un transfert de la technique à différentes catégories d'objets. Le non-transfert de la technique du façonnage au tour sur l'ensemble de la céramique suggère une différence de fonction entre les bols en " $V$ » et le reste de la céramique dont on peut supposer, qu'une partie au moins, était utilitaire. Ensuite, la présence systématique et en nombre de bols en « $V$ » en contexte funéraire ou cultuel, de même que leur association avec des céramiques dont les fonctions ne sont pas à l'évidence utilitaires (coupes à pied fenestré, cornets, vases fusiformes...) donne à cette catégorie de récipients un caractère particulier. Enfin, il apparaît peu plausible que des bols à destination domestique/utilitaire aient fait l'objet d'une importation massive $(95 \%)$ alors qu'une production locale pouvait fournir des récipients fonctionnels équivalents.

D'où l'hypothèse selon laquelle l'explication des spécificités technologiques des bols/jattes en « $V$ » est à rechercher dans leur rattachement à la sphère cultuelle, par opposition à la sphère domestique/utilitaire.

\section{LES POTIERS}

La nature des habiletés impliquées dans la fabrication des récipients élaborés au tour suggère que ces derniers étaient fabriqués par des artisans spécialisés. D'après la quantification de la production des bols élaborés au tour d'Abu Hamid, on peut supposer que ces artisans étaient peu nombreux. Ce nombre devait être d'autant plus restreint que la maîtrise d'une pratique suppose une certaine fréquence, fréquence qui ne pouvait être atteinte si la production était dispersée entre un nombre trop important de potiers. 
Les différences de qualité de façonnage pourraient être liées à des degrés de maîtrise variés de la technique. Le fait que les pièces de moindre qualité n’aient pas été détruites après leur façonnage, mais gardées, suggèrent que les différences de qualité de façonnage ne devaient pas jouer sur la valeur accordée aux récipients élaborés au tour, laquelle devait extrinsèquement tenir à la morphologie des parois et à leur transformation avec ECR.

On peut se demander dans quelle mesure ces potiers spécialisés ne pratiquaient que la technique de l'élaboration au tour, ou bien s'ils pratiquaient également la technique du façonnage sans ECR pour la fabrication d'autres types de récipients. Il est difficile de répondre à cette question dans l'état actuel de nos recherches. Des études plus fines à une échelle macro-régionale sur l'ensemble des céramiques devraient pouvoir, néanmoins, apporter des éléments de réponse. On peut seulement supposer qu'un même potier était en charge de l'ensemble du processus de fabrication des petits bols en $« V »$, le petit nombre produit $(\cong 3.5)$ rendant peu plausible l'hypothèse d'une division du travail entre ceux qui auraient façonné l'ébauche au colombin et ceux qui auraient élaboré la préforme au tour.

L'existence de potiers spécialisés dans la technique de l'élaboration au tour n'exclut pas la présence de potiers spécialisés dans la technique de façonnage sans ECR. En effet, une première approche de la céramique non tournée permet de distinguer deux qualités de productions tant sur le plan du façonnage que de la préparation des pâtes : une qualité soignée faite sur pâte finement préparée, et une qualité moins soignée faite sur pâte non décantée. A ces deux qualités de production, pourraient correspondre deux catégories d'artisans dont l'une au moins serait spécialisée.

Les habiletés impliquées dans la technique de l'élaboration au tour se seraient développées chez les artisans spécialisés ce que suggèrent à la fois la connaissance préalable des modes de préparation des pâtes et la restriction de la technique de l'élaboration au tour à une catégorie de récipients. Est-ce à dire que les artisans spécialisés qui pratiquaient le façonnage sans ECR étaient également spécialisés dans la production de récipients à valeur non domestique/utilitaire? De futures études techno-morphologiques devraient permettre de le préciser.

\section{LES RÉSEAUX DE CIRCULATION}

L'un des constats les plus étonnants que nous ait livré le site d'Abu Hamid est la très grande circulation des récipients élaborés au tour. Contre toute attente, la très grande majorité était importée et, qui plus est, du Negev. La question qui se pose alors est celle des raisons sous-jacentes à l'importation de bols qui pouvaient être. par ailleurs, fabriqués sinon sur place, du moins dans la région (ex. Pella). L'hypothèse d'une valeur cultuelle accordée à ces bols éclaire d'un jour nouveau les possibles réseaux de circulation. En effet. si la charge symbolique des bols était liée à leurs lieux de fabrication. compte tenu, par exemple, d'une hiérarchisation cultuelle des localités à une échelle macro-régionale, alors c’est le bol de la localité en question qu'il s'agissait d'acquérir et auquel ne pouvait se substituer, en aucun cas, un bol fait dans un autre lieu. Les importations seraient, dès lors motivées par des facteurs qui ne seraient pas d'ordre techno-économique, mais "identitaires», l'acquisition de bols marquant l'appartenance à une communauté cultuelle. Rappelons alors que les sites chalcolithiques du Negev, essentiellement localisés dans la partie méridionale (nahal Beer-sheva), forment un ensemble unique constitués de villages centraux, de hameaux, d'un sanctuaire, Gilat, et de nécropoles ${ }^{29}$.

Reste le problème des motivations à l'origine de l'adoption du tour. Si la valeur du récipient est prise comme explication de la motivation, alors on peut supposer que ce sont des motivations d'ordre cultuel qui sont à l'origine de l'adoption du tour. Ces motivations eurent pour conséquence, à l'échelle du Levant Sud, non seulement l'apparition d'une nouvelle technique, en rupture avec la tradition, mais également une diffusion de traits matériels sur une aire géographique large. Motivations nécessairement fortes qui témoignent, par làmême, de transformations radicales affectant la sphère cultuelle, ev/ou politico-cultuelle, à l'échelle macro-régionale, ainsi que le montre, par ailleurs, l'apparition d'aires d'inhumation spécialisées et de lieux de culte et, plus généralement, de nombreux sites d'habitat. Ces transformations peuvent être calées chronologiquement au début du $4^{\mathfrak{c}}$ millénaire avant J.-C. (niveaux supéricurs des sites chalcolithiques de la vallée du Jourdain, du Negev et des plaines côtières).

\section{CONCLUSION}

Les bols/jattes au profil en "V" des sites chalcolithiques du Sud-Levant sont souvent évoqués pour signaler l'apparition du tour en Orient. Une étude technologique, reposant sur une approche combinant lanalyse des macro-traces et des

29. I.NY et Holl. 1988. 
microfabriques, révèle d'une part la chaine opératoire de façonnage depuis le mode de préparation de l'argile jusqu'à la cuisson du récipient, d'autre part, le lieu de provenance des bols/jattes du site d'Abu Hamid. Nous retiendrons comme points essenticls que ces récipients étaient élaborés au tour, technique en rupture avec la tradition céramique locale leur conférant une qualité de "haute technicité", et qu'ils étaient, pour la grande majorité, d'origine allochtone. Les centres de production élaient variés, au scin desquels le groupe Negev qui est le plus largement représenté bien qu'étant un des plus éloignés. Il s'ensuit une séric d'hypothèses sur la valeur cultuelle des bols/jattes au profil en "V » et sur les modalités techno-économiques de production et de diffusion. En guise de conclusion, nous évoquerons les perspectives archéologiques plus générales suggérées par les transformations radicales à l'échelle macro-régionale dont témoigne l'apparition de la technique de l'élaboration au tour. Elles se rapportent aux questions sur l'organisation macro-régionale des communautés du Levant Sud: le début du $4^{e}$ millénaire atteste-t-il de sociétés structurées à l'échelle régionale ${ }^{30}$, ou, au contraire, révèle-t-il un ensemble de groupes que ne rassemble aucune forme de pouvoir, fut-il d'ordre politique ou religieux 31 ? La production des bols/jattes au profil en " $V$ » suggère qu'au début du 4 millénaire avant J.-C., les différents groupes du Sud-Levant marquaient, au travers de leur culture matérielle. leur appartenance à une même communauté cultuelle, et/ou politico-cultuelle. Cette appartenance impliquerait des réseaux de circulation d'objets à partir d'unités de production spécialisées, vraisemblablement restreintes en nombre et qui pourraient avoir été fortement liées aux autorités «cultuelles». Une élude technologique fine des céramiques non reprises au tour devrait permettre, à l'avenir, d'affiner ces hypothèses qui portent tant sur le statut des céramiques que sur les relations pouvoir/artisanat, et sur les relations verticales entre implantations à l'échelle macro-régionale.

Valentine ROUX

ERA 28 du CRA - CNRS

Maison René-Ginouvès

2I, allée de l'Université, 92023 Nanterre Cédex

Marie-Agnès COURTY

CRA - CNRS

Maison René-Ginouvès

21, allée de l'Université, 92023 Nanterre Cedex

\section{REMERCIE.MENTS}

Nous tenons ici à remercier G. Dollfus et $Z$. Kafafï pour leur aide considérable lors de l'étude du matériel d'Abu Hamid ainsi que pour leur soutien logistique. Celte étude fut achevée. dans le peu de temps qui nous était imparti. grâce à l’aide remarquable de Gabriel Humbert et de Nabil Ali sans lesquels il n'aurait pas été possible, en particulier. d'échantillonner la totalité des céramiques des niveaux supérieurs. Les lames minces ont été, en partie, réalisées dans le laboratoire du Dr Ziat el-Ahmed. par Mustapha Al Naddaf. Nous tenons ici à les remercier vivement de leur coopération. La plupart ont été faites dans le cadre de I'INA de Plaisir-Grignon. Les dessins ont été réalisés par G. Monthel (ERA 28 du CRA). L'ensemble du matériel el des traits diagnostics a été photographić par Hossein Dibajah. Nous les en remercions sincerrement.

\section{BIBLIOGRAPHIE}

BNIFIT $\mathrm{H}$.

1962 Céramiqué Ancienume alu Proche-Orient, Isrä̈l at Liban. VI'-III' millénaire. Étude technique. Paris: Dockorat is-lettres.

CoMnen:Nit:-Pl:III:RIN C.

1987 La poterie d`Abou Matar el de l'ouadi \%oumeili (Beershéval) au IV" millénaire avant l'ère chrétienne. Le's Cahie'ss du C'entre" de Recherche Français de Jérusale'm 3. Paris: Association Palcórient.

1990 La poterie de Safadi (Beershéva) au IV" millénaire avant lère chrétienne. Le's Cahie'ss du Centre de recherche Franģais de Jérusalem 5. Paris: Association Paléorient.

Col RTY M. A. and Rol $x \mathrm{~V}$.

1995 Identification of wheel throwing on the basis of ceramic surface features and microtabrics. Jominal of Archaeselengical Science 22: $17-50$.

DOBRE:S M. A. and Hor NMN C.R.

1994 Social Agency and the Dynamics of Prehistoric Technology. Journal of Archaeological Method and Theory $1: 211-258$.

D)I.L.'S G. el KAFAl $Z$.

1988 Abu Hamid. un jalon dans le temps et dans lespace. In : Abu Hamid. village du $4^{r}$ millénaire de la vallée du Jomedain: 51-52. Amman (Jordanic) : Economic press.

ERICSON K.A. and I.I:MMA A.C:

1996 Expert and exceptionnal performance: evidence of maximal adaptation (o) task constraints. Ammal Review of Psychology $47: 27,3-30.5$.

GI:I.I3I:Ri $A$.

sous presse De l'élaboration au tour au tournage sur motte: difficultés motrices el conceptuelles. Technique's at Culture.

GILI:AI) !.

1988 The Chalcolithic period in the Levant. Joturnal of World Pre histom $2: 397-443$.

van der l.1:H:W S.I:

1994 The pottery from a Middle-Lruk pit at Tepe Sharafabad. Iran. A lechnological study. In : Terre cuite et Société. La céramique, document technique, écomomique, culturel: 269-302. XIV" Rencontres Internationales d'Archéologic et d'Histoire d'Antibes. Juan-les-Pins: Iditions APICCA.
30. Ihid.

31. Gillith), 1988. 


\author{
L.MIONNIER P. (ed.) \\ 1993 Technological rhoices: tramsformation in material cultures \\ since the Neolithic. Iondon and New York: Routledge.
}

L.H.Y T.E:

1985 Shiqmim: a chalcolithic village and mortuary centre in the Vorthern Negev desert. Paléorient II: $71-8.3$.

L.iv T.I. et Hol.L. $A$.

1988 Les sociétés chalcolithiques de la Palestine et l'émergence de chefferies. Archives Européennes de Sociologie XXIX : 28.3316.

LEYY T.E. and MiNAlli: N.

1987 The Ceramic Industry at Shicmim: typological and spatial considerations. In : LI:VY T.E. Shiqmim I. Studies concerning Chalcolithic Societies in the northern Negev Desert. Israel (1982-1984). BAR lmt. Ser. 356. Oxford.

MAYORA.

1994 Durées de vie des céramiques africaines : facteurs responsables et implications archéologiques. In : Terre crite et Société. La

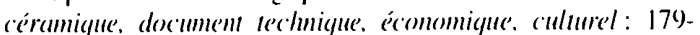
198. XIV“ Rencontres Internationales d'Archéologie et d'Hisloire d'Antibes. Juan-les-Pins: liditions APDCA.

ORION C.. TYliks P. and VINCI: A.

1993 Pottery in archaeolog!: Cambridge: Cambridge Lniversity Press.

Pirkot J. Cl Ladiray D.

1980 Tombes à ossuaires de la région côtière palestinienne au IV" millénaire avant l'ère chrétienne. MTJ I Paris: Association Paléorient.

PII:RRI:I A.

1995 Analise terhnologique des céramiques ardhélogiques: développements méthodologique's pour lidentification des terhniques de fareonnagere. Un exermple d'application: le matériel du villege des Arines is lovrome (Indre). Paris I, PanthéonSorbonne: These de Doctorat.

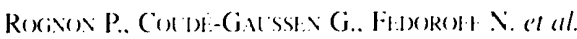

$1987 \quad$ Micromorphology of loess in the Northern Neges (Isacl). In:

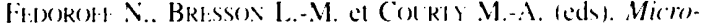
morphologie des Sols: 6.31-6.38. Plaisir. Hrance : AlES.

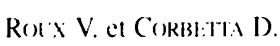

1990 Technique du tournage es spécialisation artisanale. In: Ron'X

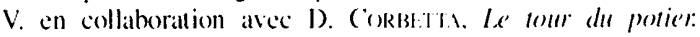
Spécialisation artisanale et compétences techmiques: 19-99. Monographic du CRA n" +. Paris : Éditions du C.NRS.

Rolx V. and CorkTY M. A.

sous presse Identification of whecl fashioning methods: a methodological tool for technological analysis of th-3rd millenium B.C. Asian ceramics. Journal of Archaesological Sicionce.

Rox V. et MATARASSO P.

sous presse (rafts and the evolution of complex societies: new perspeclives for modeling the organization of production - An Hatrappan example - $I n$ : I)(OBRES M.A. and HOIFMAN C.R. Making Colture : Exsays on Tédmological Practice. Politics and world viens. Washington: Smithsonian Institution Press.

Rrit ().S. (ed.)

1981 Pottery Techmolog!: Principles and Reconstruction. Washington D.C. : Taraxacum Press.

(jssisikix D).

1980 The Ghassulian temple al In-ciedi. Tel Awiv VII : 1-H.

V:III.I.ANT N.

à paraître La céramique des niveaux supérieurs. Typologice. In : Dentrots

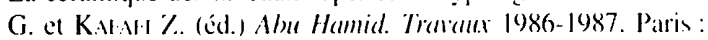
I.R.C.

YANo(N 1).H. and DAN J.

1974 Accumulation and distribution of loess-derived deposits in the semi-desert and desert fringe areas of lsratel. Z. (jeomorphl. N.I: Suppl. Bd. 20: 91-105. 